\title{
WIP: What Makes Courses Demanding in Engineering Education? A Combination of Mixed Methods and Grounded Theory Research
}

\author{
Miss Isabel Hilliger P.E., Pontificia Universidad Catholica de Chile
}

Isabel Hilliger is the Associate Director for Assessment and Evaluation at the Engineering Education Division in Pontificia Universidad Católica de Chile (UC). Isabel received a BEng from UC and an MA in Education Policy from Stanford University. She is currently a PhD Candidate in Computer Science at UC-Engineering. Her research theme is the use of methodologies and analytical tools for continuous curriculum improvement in Higher Education. She has created qualitative and quantitative instruments for outcome assessment in enginering education. She has also evaluated policy efforts towards engineering diversity and undergraduate research.

\section{Miss Constanza Melian, Pontificia Universidad Católica de Chile}

Sociologist. Assessment and Evaluation Coordinator, Division of Engineering Education (Pontificia Universidad Católica de Chile).

\section{Miss Javiera Meza, Pontificia Universidad Católica de Chile}

Javiera Meza has a Bachelor of Engineering Science in Pontificia Universidad Católica de Chile. She is a research assistant of the Engineering Education Division, responsible for supporting research tasks and collaborating in data collection and analysis. Javiera developed a project about STEM education focused on primary school. Her research theme is about gender gap and motivation of students in undergraduate computer science programs. Currently she is researching about student motivation in online lessons due to the influence of COVID-19.

\section{Mr. Gonzalo Cortés, Pontificia Universidad Católica de Chile}

Gonzalo Cortés is an undergraduate student at the engineering school in Pontificia Universidad Católica de Chile. His Major is Electrical Engineering and his Minor is Energy. Currently, he is a research assistant of the Engineering Education Division, responsible for supporting research tasks and collaborating in data collection and analysis. Gonzalo managed a pre-engineering program to encourage high school students to study careers in engineering and science. He also volunteered as a teacher in communication skills and personal development, aimed at training high school students in vulnerable backgrounds.

\section{Dr. Jorge A Baier, Pontificia Universidad Catholica de Chile}

Is an associate professor in the Computer Science Department and Associate Dean for Engineering Education at the Engineering School in Pontificia Universidad Católica de Chile. Jorge holds a PhD in Computer Science from the University of Toronto in Canada and a Master's Degree in Engineering Sciences from Pontificia Universidad Católica de Chile. His research focuses on areas of automated reasoning in Artificial Intelligence; specifically, automated planning, search and knowledge representation. Currently his research focuses on understanding how machine learning techniques can be applied to the intelligent decision-making process, on the applicability of reasoning techniques and learning to databases. He is also an assistant researcher at the Millennium Institute for Foundational Research on Data. 


\section{WIP: What Makes Courses Demanding in Engineering Education? A Combination of Mixed Methods and Grounded Theory Research}

\section{Introduction}

Engineering undergraduate programs have become demanding in terms of workload [1]. Along with class time schedules packed with lectures, laboratories, and tutorials, there are a significant number of course assignments that occur outside of class, such as team-based projects and experiential learning tasks [1]. Researchers have encouraged the incorporation of these constructivist approaches into engineering education [2], aiming to help students develop a wide range of abilities (such as complex-problem solving skills and interdisciplinary thinking [3]). However, this increasing number of assignments stresses students [4], [5], negatively affecting their learning results [1], [6].

To understand what students define as a demanding course, several researchers have explored the concepts of academic workload and course difficulty [1], [4]-[7]. So far, there is a growing body of knowledge in Canada and the U.S. regarding factors that affect how first-year students perceive workload [1]. However, little is known about how students perceive course difficulty after dealing with their transition from high school to college, and how the quality of teaching affects their approach to learning [6]. Thus, not only more studies are needed to understand how student-centered approaches could enrich learning experiences from a multi-dimensional perspective [1], [3], [4], but also to examine how these multidimensional approaches make learning more meaningful at a course level [4]. This is particularly relevant in Chile, considering that previous studies have demonstrated that students who major in science and engineering often use surface approaches to learning, focusing on course content that they believe they must memorize to meet assessment requirements [8].

This paper presents a Work-In-Progress (WIP) that is part of a larger study to understand students' perceptions on engineering courses imparted at Pontificia Universidad Católica de Chile (PUC-Chile). The research question addressed in this paper is: What factors affect students ' perceptions on demanding courses in terms of difficulty? To answer this research question, we combined mixed methods with grounded theory research (MM-GT). By MMGT, we mean the systematic collection and integration of both qualitative and quantitative data toward the goal of theory development [9]. According to recent studies, the MM-GT research approach has become useful to develop and test theory in the fields of education [8], [9]. In this study, we plan to develop theoretical models of difficulty at a course level, following best practices of MM-GT application to provide insights for course curriculum development and teaching reflection in the field of engineering education.

\section{Research Design and Current Data Collection}

In this study, we plan to use an exploratory sequential design based on MM-GT to develop and test theoretical models in four phases (see Figure 1). This paper presents the results of the first phase, which consisted of a grounded theory approach to identify the factors associated to what students perceive as easy courses and difficult courses. In the second 
phase, we plan to integrate the factors identified in the first phase into an online survey with multiple-response questions, aiming to measure how predominant are these factors in a larger population of engineering students. Then, in the third phase, this online survey will be used to collect data at the same engineering school were the focus groups were conducted. Finally, the fourth phase of the study will integrate the quantitative results of the survey with the grounded theory model to develop a theory that more accurately describes how different factors influence students' perspectives on course difficulty, besides revealing whether these factors are associated to meaningful learning.

\begin{tabular}{|c|c|c|c|}
\hline $\begin{array}{l}\text { Qualitative Phase } \\
\text { Grounded Theory }\end{array}$ & $\begin{array}{r}\text { Integ } \\
\text { Surve }\end{array}$ & $\begin{array}{c}\text { Quantitative Phase } \\
\text { Survey Study }\end{array}$ & $\begin{array}{l}\text { Integration Phase } \\
\text { Synthesis }\end{array}$ \\
\hline $\begin{array}{l}\text { Purposive sample of } \\
+20 \text { engineering } \\
\text { students } \\
\text { Data collection through } \\
\text { focus groups } \\
\text { Selective coding for } \\
\text { model development }\end{array}$ & $\begin{array}{l}\text { - Develop survey with } \\
\text { multiple-response } \\
\text { questions } \\
\text { - Refine survey: } \\
\text { - Review by expert } \\
\text { - Cognitive interviews }\end{array}$ & $\begin{array}{l}\text { - Enumerative sample of } \\
+1.000 \text { engineering } \\
\text { students } \\
\text { - Data collection through } \\
\text { online survey } \\
\text { - Tabulation of mutiple } \\
\text { responses }\end{array}$ & $\begin{array}{l}\text { - Refine grounded theory } \\
\text { model based on survey } \\
\text { results } \\
\text { - Identify lessons learned }\end{array}$ \\
\hline
\end{tabular}

Figure 1. Mixed methods-grounded theory exploratory sequential design

During the first phase of this study, we conducted two focus groups with a purposive sample of engineering undergraduate students. By a purposive sample, we mean a selection of study participants from a particular section of the population of interest [10], a sampling strategy widely used in social sciences and other fields [10]-[12]. Considering our interest in students' perception on course difficulty and meaningfulness, we selected engineering students in their second year of study or older, aiming to explore factors that are associated to course design (course-related factors), faculty characteristics and influences (facultyrelated factors), or student characteristics (students related-factors), and not necessarily associated to their transition from high-school to first-year engineering [4]).

A total number of 23 students participated in each focus group: 13 students in Focus Group 1 and 10 in Focus Group 2. In both samples, there were students from different admission cohorts (2018, 2017, 2016, 2015 and 2014) and different engineering programs (Biological Engineering, Biomedical Engineering, Chemical Engineering, Civil Engineering, Electrical Engineering, Engineering Design and Innovation, Industrial Engineering, Mathematical Engineering, and Mechanical Engineering). Both focus groups followed a semi-structured protocol based on previous work on academic rigor [7] (see protocol here: http://bit.ly/3aWGM8c). Students were interviewed in a 1-hour session under informed consent, and the audio files obtained from both focus-groups were transcribed verbatim. The analysis was conducted by three researchers, who discussed initial and selective codes to identify emerging factors for what students perceive as easy courses, difficult courses, and meaningful courses in terms of learning. Memos were written to record the description and evolution of codes (see final coding scheme here: http://bit.ly/36th6ww), and theoretical models were developed concurrently with the coding process. 


\section{Preliminary Results}

We obtained one grounded theory model from coding the focus groups transcripts, which describes the factors associated to engineering students' perceptions on course difficulty, distinguishing among course-related, faculty-related, and student-related factors (see Figure 2). Regarding course-related factors, students mentioned the complexity and the amount of content, the constructive alignment of course teaching and assessment methods and the amount of workload, besides their perceptions on how the course content and activities are relevant for real life applications. Concerning faculty-related factors, students alluded to the willingness of faculty to provide support and guidance to students, in addition to faculty teaching skills to explain complex content and to plan learning-oriented classes. Finally, among student-related factors, students indicated that their perceptions are also influenced on their prior knowledge and their individual interest on course content and activities.

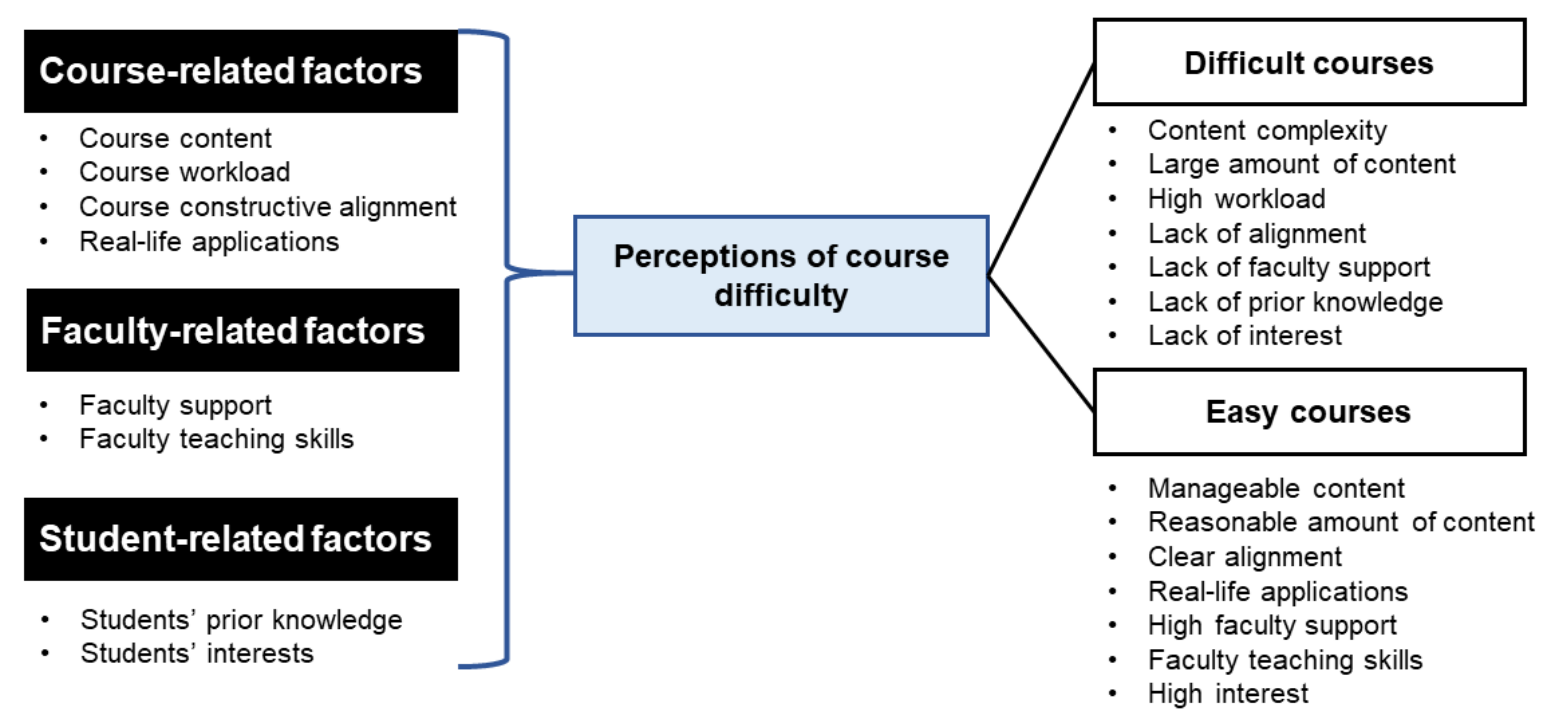

Figure 2. Grounded theory model to describe factors associated to difficult and easy courses from the perspective of engineering students (see details of the coding analysis in http://bit.ly/36th6ww)

Table 1. Additional factors associated with meaningful learning in courses perceived as difficult or easy (see details of the coding analysis in http://bit.ly/36th6ww)

\begin{tabular}{ll}
\hline & Additional factors \\
\hline Course-related factors & - Course learning opportunities \\
& - Real-life applications \\
Faculty-related factors & - Faculty motivation for teaching \\
& - Faculty support \\
Student-related factors & - Faculty teaching skills \\
& - Students' motivation \\
\hline
\end{tabular}


From the information obtained from the interviews, we also identified additional factors that are associated with meaningful learning in courses perceived as difficult or easy (see Table 1). Regardless of the difficulty of a course, students perceive a course as meaningful if it includes learning opportunities to apply content and to understand its relevance regarding real-life applications. Concerning faculty-related factors, students alluded to the importance of the intrinsic motivation of faculty members for teaching, in addition to the relevance of their capacities to provide support and to teach complex content (particularly relevant in difficult courses). Finally, among student-related factors, students indicated that their learning is influenced by their motivation and interests in course content and activities.

\section{Discussion, Limitations, and Future Work}

Our preliminary results suggest that course difficulty is a multidimensional construct. Regarding difficult and easy courses, students mentioned the amount and complexity of course content, the amount of course workload, and the alignment between course teaching assessment methods. The first two factors have also been mentioned in previous studies, whose results indicate that students' perceptions are influenced by the perceived complexity of course content and the amount of work required week-to-week [1]. However, there is less evidence regarding constructive alignment of course elements, so more studies are needed to provide teaching staff with guidance on how to incorporate constructivist approaches to learning [2], [3].

Our preliminary results also suggest that there are additional factors that are associated with meaningful learning regardless of course difficulty. Students mentioned that learning opportunities to apply content in practical projects are relevant in any type of course, in addition to class activities that allow them to apply theoretical concepts in real-world applications. They also mentioned the importance of their motivation and interests in course content and activities, besides alluding to faculty motivation and teaching skills. This finding resonates with prior studies that associate student learning with active teaching, learning motivation, and content complexity [2], [6]. However, this finding also opens up an opportunity for further studies to understand how these additional factors could help teaching staff manage course difficulty and students' motivation in engineering coursework.

So far, this study has been conducted with a small population at the engineering school in PUC-Chile, which implies potential generalization issues. To address this limitation, future work includes collecting quantitative data from a larger population of students. These data will be used to explore what predominant factors influence students' perceptions on course difficulty in the light of institutional factors and educational theories. Still, further studies should also consider exploring perceived course difficulty in engineering schools located in other countries, in order to discuss implications for different educational systems.

\section{Acknowledgments}

This work was supported by CORFO under grant no. 14EN12-26862.The authors would like to thank Paolo Fabia, Angela Parra, and Sebastián Vásquez for motivating this study as student representatives in 2019, aiming to create a shared meaning for course demand among students, teaching staff, and managers. 


\section{References}

[1] D. Gerrard, K. Newfield, N. B. Asli, and C. Variawa, "Are students overworked? Understanding the workload expectations and realities of first-year engineering," in ASEE Annual Conference and Exposition, 2017.

[2] M. Christie and E. de Graaff, "The philosophical and pedagogical underpinnings of Active Learning in Engineering Education," Eur. J. Eng. Educ., vol. 42, no. 1, pp. 516, 2017.

[3] E. J. H. Spelt, P. A. Luning, M. A. J. S. van Boekel, and M. Mulder, “A multidimensional approach to examine student interdisciplinary learning in science and engineering in higher education," Eur. J. Eng. Educ., vol. 42, no. 6, pp. 761$774,2017$.

[4] D. Gerrard and C. Variawa, "Bridges and barriers: A multi-year study of workloadrelated learning experiences from diverse student and instructor perspectives in firstyear engineering education," in ASEE Annual Conference and Exposition, 2018.

[5] T. Geyer and W. R. Loendorf, "Handling increased faculty and student workload during difficult economic times," in ASEE Annual Conference and Exposition, 2015.

[6] H. P. Andres, "Active teaching to manage course difficulty and learning motivation," J. Furth. High. Educ., vol. 43, no. 2, pp. 220-235, 2019.

[7] K. Bowyer, "A model of student workload," J. High. Educ. Policy Manag., vol. 34, no. 3, pp. 239-258, 2012.

[8] H. Yin, C. González, and S. Huang, 'Undergraduate students' approaches to studying and perceptions of learning context: a comparison between China and Chile," High. Educ. Res. Dev., vol. 37, no. 7, pp. 1530-1544, 2018.

[9] M. C. Howell Smith, W. A. Babchuk, J. Stevens, A. L. Garrett, S. C. Wang, and T. C. Guetterman, "Modeling the Use of Mixed Methods-Grounded Theory: Developing Scales for a New Measurement Model," J. Mix. Methods Res., 2019.

[10] J. M. Guarte and E. B. Barrios, "Estimation Under Purposive Sampling," Commun. Stat. - Simul. Comput., vol. 35, no. 2, pp. 277-284, 2007.

[11] J. O. Keeffe, W. Buytaert, A. Mijic, and N. Brozovi, "The use of semi-structured interviews for the characterisation of farmer irrigation practices," Hydrol. Earth Syst. Sci., vol. 20, pp. 1911-1924, 2016.

[12] M. D. C. Tongco, "Purposive Sampling as a Tool for Informant Selection," Ethnobot. Res. Appl., vol. 5, pp. 147-158, 2007 